\title{
Early Acceleration of COVID-19 in Areas with Larger Nursing Homes and Certificate of Need Laws
}

\author{
Cyrus M. Kosar, MA ${ }^{1,2}$ and Momotazur Rahman, $P h D^{1,2}{ }_{\infty}$ \\ 'Department of Health Services, Policy \& Practice, Brown University School of Public Health, Providence, RI, USA; ${ }^{2}$ Center for Gerontology and \\ Healthcare Research, Brown University, Providence, RI, USA.
}

\begin{abstract}
BACKGROUND: Severe acute respiratory syndrome coronavirus 2 (SARS-CoV-2) outbreaks have become common in large nursing homes, placing not only residents but also staff and community members at risk for infection. However, the relationship between larger nursing homes and the community spread of SARS-CoV-2 has not yet been documented.
\end{abstract}

OBJECTIVE: To examine the association between county average nursing home bed size and presence of certificate of need (CON) laws, which influence nursing home size, with county-level SARS-CoV-2 prevalence over time.

DESIGN: Cross-sectional study using county-level data from March 11 through June 12, 2020.

PARTICIPANTS: All US counties with at least one nursing home $(n=2,883)$.

MAIN MEASURES: The main explanatory variables were county average nursing home bed size and presence of a CON law. The main outcome was the cumulative number of SARS-CoV-2 cases on each day of the study period adjusted for county population size and density, demographic and socioeconomic characteristics, total nursing home bed supply, other health care supply measures, epidemic stage, and census region.

KEY RESULTS: By June 12, a between-county difference in average nursing home size equal to 1 bed was associated with 3.92 additional SARS-COV-2 cases $(95 \% \mathrm{CI}=2.14$ to 5.69; $P<0.001$ ), on average, and counties subject to CON laws had 104.53 additional SARS-CoV-2 cases $(95 \% \mathrm{CI}=$ 7.68 to $201.38 ; P<0.05)$, on average. Counties with larger nursing homes also demonstrated higher growth in the frequency of SARS-COV-2 throughout the study period.

CONCLUSIONS: At the county level, average nursing home size and CON law presence was associated with a greater frequency of SARS-CoV-2 cases. Controlling the impact of the coronavirus 2019 pandemic may require additional resources for communities with larger nursing homes and more attention towards long-term care policies.

KEY WORDS: COVID-19; nursing homes; long-term care; community spread; certificate of need law.

J Gen Intern Med 36(4):990-7

DOI: $10.1007 / \mathrm{s} 11606-020-06518-2$

(C) Society of General Internal Medicine 2021

Prior Presentations None.

Received June 30, 2020

Accepted December 20, 2020

Published online January 28, 2021

\section{INTRODUCTION}

At the time this report was written, the severe acute respiratory syndrome coronavirus 2 (SARS-CoV-2) had infected over 10 million individuals worldwide and caused over 500,000 deaths. ${ }^{1}$ In the United States (US), the coronavirus disease 2019 (COVID-19) pandemic has disproportionately impacted communities of color and vulnerable populations such as the frail elderly. ${ }^{2-4}$ Indeed, the majority of this country's nursing homes have had SARS-CoV-2 outbreaks. ${ }^{5}$ Several studies have found that nursing home outbreaks were unrelated to traditional measures of nursing home quality and were instead associated with facility size and location. ${ }^{6,7}$

Local SARS-CoV-2 prevalence is likely to have a strong impact on nursing home infection rates. At the same time, nursing homes may contribute to the community spread of SARS-CoV-2 for several reasons. A large fraction of nursing home residents occupy shared bedrooms and cannot be isolated from one another. In addition, most residents require physical assistance with activities of daily living and therefore frequent contact with direct care staff. Furthering the risk of transmission, low-wage direct care staff commonly hold multiple jobs, sometimes at multiple nursing homes. Additionally, in a survey of almost 1,000 nursing home workers, 70\% reported feeling obligated to work while sick. ${ }^{8}$

All of these circumstances may not only increase SARSCoV-2 infection risk among nursing home residents, but also may accelerate SARS-CoV-2 infection rates in the communities where facilities, particularly those of larger size, are located. Communities with larger nursing homes may be more at risk simply because there is exposure between greater numbers of residents, staff, and outside visitors prior to the adoption of admission bans. However, the relationship between the presence of larger nursing homes and the community spread of SARS-CoV-2 has not yet been documented.

A nursing home's size is perceptibly connected to local population density. However, federal and state policies are potential contributing factors. For example, in some states, Medicaid reimbursement and staffing levels are tied to bed size thresholds. Under federal law, nursing homes with 121 or more beds are required to staff a licensed social worker full time. ${ }^{9}$ certificate of need (CON) laws, another state-level policy, also indirectly impact nursing home size through placing a ceiling on the supply of nursing home beds. As shown in 
previous studies, CON laws result in larger nursing homes in the long run due to the substantial fixed (capital) cost of proving long-term care. Specifically, in the presence of $\mathrm{CON}$, the higher average fixed cost per bed for smaller facilities cannot be offset with the addition of some needed number of revenue-generating beds. Over time, this has resulted in the presence of fewer nursing home operators and existing nursing homes becoming larger in states with $\mathrm{CON} .{ }^{10,11}$

In this study, we examined the association between the average nursing home bed sizes of counties with its SARSCoV-2 prevalence from March 11, 2020, the date the SARSCoV-2 outbreak was declared a pandemic, through June 12, 2020. Importantly, the earlier portion of the study period includes the time when the majority of nursing homes were not reporting outbreak data publicly. We also compared SARS-CoV-2 prevalence between counties located in states with and without nursing home $\mathrm{CON}$ laws due to their indirect impact on facility size.

\section{METHODS}

This study did not require Institutional Review Board oversight as all of the data sources are publicly available, aggregate data.

\section{Study Population}

The units of analysis for this study were US counties. All counties with nursing homes were included except for the five containing the boroughs of New York City and three containing the Kansas City municipality because COVID-19 data were reported in aggregate for these areas.

\section{Data Sources}

County population characteristics were obtained from the 2018-2019 Area Health Resources file (AHRF). ${ }^{12}$ The county-level AHRF was created by the Health Resources and Services Administration and consists of data from several public sources such as the Census. The AHRF includes county demographic composition, measures of health care supply and population health, and multiple socioeconomic indicators. Daily COVID-19 data were gathered from a publicly available repository maintained by John Hopkins University's Center of Systems Science and Engineering (CCSE). ${ }^{13}$ The CCSE collects data on SARS-CoV-2 infections and related deaths reported by county and state public health departments. Data are unified into a county-level dataset containing cumulative SARS-CoV-2 infections and deaths on each day since January 1, 2020.

Nursing home- and hospital-level data were obtained from the current Certification and Survey Provider Enhanced Reporting database (nursing home annual surveys) and Medicare Provider of Services files, respectively, and aggregated to the county level. National Conference of State Legislature data was used to determine state nursing home CON law status.

\section{Outcomes}

The primary outcome of this study was the cumulative number of SARS-CoV-2 cases on each day between March 11 and June 12, 2020. Consistent with contemporaneous work, we controlled for population size in regression analyses (discussed below), which facilities a flexible analytic approach compared with examining cases per capita directly. ${ }^{14}$ For some analyses, we focused on data from the last day of the study period alone. We also examined SARS-CoV-2-related deaths as a secondary outcome.

\section{Explanatory Variables}

The main explanatory variables were the average nursing home bed size of a county and whether or not the county was located in a state with a nursing home CON law. For some analyses, we classified county average nursing home size as small, medium, and large if the average number of beds across nursing homes was equal to below 90 beds, 91 to 120 beds, and greater than 121 beds, respectively.

\section{Statistical Analysis}

In unadjusted comparisons, we examined differences in the characteristics of counties according to average nursing home size level. We also assessed differences in the average size of nursing homes between counties located in states with and without CON laws conditional on population density level (persons per square land mile), which is likely to be a key determinant of nursing home size. We then estimated the adjusted association between average nursing home size and the cumulative number of SARS-CoV-2 cases on June 12, the last day of the study period, using Poisson regression. To facilitate interpretation, we presented the marginal effect of average nursing home size on the number of cases, interpreted as the adjusted difference in SARS-CoV-2 case count associated with a between-county difference in average nursing home size equal to one bed.

From the Poisson model, we derived adjusted SARS-CoV2 prevalence at the state level to compare the relationship between average nursing home size and SARS-CoV-2 prevalence for individual states with and without $\mathrm{CON}$ laws. In a separate model, we examined the relationship between CON law and SARS-CoV-2 case count more directly by supplanting average nursing home size with state $\mathrm{CON}$ law as the explanatory variable of interest. We also examined whether growth in SARS-CoV-2 cases varied with average nursing home size (classified as small, medium, or large) and CON laws by fitting separate regression models for each day of the study period.

Multivariable models were adjusted for large set of covariates that may be related to the presence of larger nursing 
homes and COVID-related outcomes (Table 1). In brief, these were population size and density, demographic composition, socioeconomic indicators, nursing home bed supply, and other health care supply measures. To capture population density and regional variation further, we also controlled for the number of housing units, census region, and an urban versus rural county indicator. ${ }^{15}$ We also controlled for days since the county's first SARS-CoV-2 case - a proxy for epidemic stage. The analysis of death included the number of cases as an additional covariate. Adjusted predictions and marginal effects were derived using the marginal standardization form of predictive margins. All regression models included robust standard errors. Data were analyzed with Stata MP 16.0 (StataCorp, College Station, TX). Null hypotheses were tested assuming a two-sided type I error probability of 0.05 .

\section{Sensitivity Analyses}

As a robustness check, we excluded counties located in New York and New Jersey, the states most severely impacted by COVID-19 during the study time period, from our main analysis. We also estimated the adjusted association between three alternative primary explanatory variables and the number of SARS-CoV-2 cases. These were average nursing home size based on number of residents (occupied beds), median nursing home size, and an indicator for whether a county had at least one large ( $\geq 121$ bed) nursing home (which are prevalent in over $40 \%$ of counties with nursing homes). In another alternative specification, we included both primary explanatory variables, average nursing home size, and CON status, as well as their interaction.

\section{RESULTS}

The characteristics of the 2,883 counties in our sample, stratified by average nursing home size, are presented in Table 1 . Counties with larger nursing homes had larger populations sizes, were more densely populated, more likely to be metropolitan, and located in the South or Northeast. In terms of demographic and socioeconomic characteristics, counties with larger nursing homes had a higher share of Black residents, a lower share of individuals aged 65 and over, a higher share of college-educated residents, and had a higher median household income. Health care supply, including the number of nursing home beds, hospital beds, home health agencies, and physicians was higher in counties with larger nursing homes.

The relationship between nursing home size and CON laws is depicted in Fig. 1. Panel A shows differences in average nursing home size between counties located in states with and without CON laws conditional on population density level. Panel B shows the proportion of all nursing home beds in a county that are located in medium and large ( $\geq 90$ bed) versus small $(<90$ bed) facilities for counties subject to and not subject to CON conditional on population density level. The average size of nursing homes was larger in counties affected
Table 1 United States County Characteristics by Average Nursing Home Size

\begin{tabular}{|c|c|c|c|c|}
\hline \multirow[b]{2}{*}{ Characteristic } & \multicolumn{3}{|c|}{ Average nursing home size of county } & \multirow[b]{2}{*}{$P^{*}$} \\
\hline & $\begin{array}{l}\text { Small } \\
\text { No. beds } \\
<90(n= \\
1361)\end{array}$ & $\begin{array}{l}\text { Medium } \\
90-120 \\
\text { beds }(n= \\
1057)\end{array}$ & $\begin{array}{l}\text { Large } \\
\text { No. beds } \\
\geq 121(n= \\
465)\end{array}$ & \\
\hline $\begin{array}{l}\text { Population size } \\
(1000 \mathrm{~s}) \text {, mean (sd) }\end{array}$ & $\begin{array}{l}36.8 \\
(83.8)\end{array}$ & $\begin{array}{l}157.7 \\
(461.5)\end{array}$ & $\begin{array}{l}212.8 \\
(421.2)\end{array}$ & $\begin{array}{l}< \\
0.001\end{array}$ \\
\hline $\begin{array}{l}\text { Population density, } \\
\text { mean (sd) } \\
\text { Census region, no. } \\
(\%)\end{array}$ & $\begin{array}{l}61.6 \\
(158.6)\end{array}$ & $\begin{array}{l}252.6 \\
(633.2)\end{array}$ & $\begin{array}{l}665.8 \\
(1723.8)\end{array}$ & $\begin{array}{l}< \\
0.001 \\
< \\
0.001\end{array}$ \\
\hline Northeast & $37(3)$ & $80(8)$ & $91(20)$ & \\
\hline Midwest & 679 (50) & $263(25)$ & $52(11)$ & \\
\hline South & 435 (32) & 601 (57) & $302(65)$ & \\
\hline West & $210(15)$ & 113 (11) & $20(4)$ & \\
\hline $\begin{array}{l}\text { Metropolitan } \\
\text { county, no. }(\%)\end{array}$ & $312(23)$ & $516(49)$ & $282(61)$ & $\begin{array}{l}< \\
0.001\end{array}$ \\
\hline $\begin{array}{l}\text { Population } \% \text { aged } \\
\geq 65 \text { years, mean } \\
\text { (sd) }\end{array}$ & $19.5(4.3)$ & $18.0(4.3)$ & $17.4(3.9)$ & $\begin{array}{l}< \\
0.001\end{array}$ \\
\hline $\begin{array}{l}\text { Population \% } \\
\text { female, mean (sd) }\end{array}$ & $49.7(2.4)$ & $50.1(2.1)$ & $50.5(2.1)$ & $\begin{array}{l}< \\
0.001\end{array}$ \\
\hline $\begin{array}{l}\text { Population \% } \\
\text { Black, mean (sd) }\end{array}$ & $6.3(13.4)$ & $11.0(14.5)$ & $\begin{array}{l}16.1 \\
(16.3)\end{array}$ & $\begin{array}{l}< \\
0.001\end{array}$ \\
\hline $\begin{array}{l}\text { Population } \% \text { with/ } \\
\text { without college de- } \\
\text { gree, mean (sd) }\end{array}$ & $19.9(7.9)$ & $21.6(9.7)$ & $\begin{array}{l}23.9 \\
(10.6)\end{array}$ & $\begin{array}{l}< \\
0.001\end{array}$ \\
\hline $\begin{array}{l}\text { Median household } \\
\text { income, mean (sd) }\end{array}$ & $48.9(11)$ & $49.7(13.9)$ & $\begin{array}{l}51.9 \\
(15.9)\end{array}$ & $\begin{array}{l}< \\
0.001\end{array}$ \\
\hline $\begin{array}{l}\text { Poverty rate, mean } \\
\text { (sd) }\end{array}$ & $15.5(6.6)$ & $16.7(6.2)$ & $16.5(6.0)$ & $\begin{array}{l}< \\
0.001\end{array}$ \\
\hline $\begin{array}{l}\text { Nursing home bed } \\
\text { supply, mean (sd) }\end{array}$ & $\begin{array}{l}218.4 \\
(306.5)\end{array}$ & $\begin{array}{l}747.1 \\
(1683.1)\end{array}$ & $\begin{array}{l}1126.4 \\
(2231.9)\end{array}$ & $\begin{array}{l}< \\
0.001\end{array}$ \\
\hline $\begin{array}{l}\text { Nursing home } \\
\text { certificate of need } \\
\text { law, no. }(\%)\end{array}$ & $847(62)$ & $785(74)$ & $383(83)$ & $\begin{array}{l}< \\
0.001\end{array}$ \\
\hline $\begin{array}{l}\text { Small hospital bed } \\
\text { supply, mean (sd) }\end{array}$ & $\begin{array}{l}62.5 \\
(54.0)\end{array}$ & $\begin{array}{l}82.8 \\
(133.8)\end{array}$ & $\begin{array}{l}69.7 \\
(108.8)\end{array}$ & $\begin{array}{l}< \\
0.001\end{array}$ \\
\hline $\begin{array}{l}\text { Average-sized } \\
\text { hospital bed supply, } \\
\text { mean (sd) }\end{array}$ & $\begin{array}{l}46 \\
(158.9)\end{array}$ & $\begin{array}{l}262.9 \\
(923.0)\end{array}$ & $\begin{array}{l}404.3 \\
(904.0)\end{array}$ & $\begin{array}{l}< \\
0.001\end{array}$ \\
\hline $\begin{array}{l}\text { Large hospital bed } \\
\text { supply, mean (sd) }\end{array}$ & $\begin{array}{l}24.1 \\
(175.6)\end{array}$ & $\begin{array}{l}183.4 \\
(748.6)\end{array}$ & $\begin{array}{l}368.8 \\
(1161.4)\end{array}$ & $\begin{array}{l}< \\
0.001\end{array}$ \\
\hline $\begin{array}{l}\text { No. home health } \\
\text { agencies, mean (sd) }\end{array}$ & $1.1(2.7)$ & $6.0(30.9)$ & $7.6(32.8)$ & $\begin{array}{l}<. \\
0.001\end{array}$ \\
\hline $\begin{array}{l}\text { No. medical doctors } \\
\text { (per 1000), mean } \\
\text { (sd) }\end{array}$ & $0.1(0.3)$ & $0.4(1.6)$ & $0.7(1.8)$ & $\begin{array}{l}< \\
0.001\end{array}$ \\
\hline $\begin{array}{l}\text { No. housing units } \\
\text { (per 1000), mean } \\
\text { (sd) }\end{array}$ & $\begin{array}{l}16.4 \\
(32.4)\end{array}$ & $\begin{array}{l}64.8 \\
(170.3)\end{array}$ & $\begin{array}{l}89.4 \\
(172.4)\end{array}$ & $\begin{array}{l}< \\
0.001\end{array}$ \\
\hline
\end{tabular}

No., number of. Population density is equal to the number of persons per square mile of land. Small-, medium-, and large-sized hospitals denote those with, $<100,100$ to 399, and $\geq 400$ beds, respectively *Derived from a one-way ANOVA for continuous variables or chisquared test for categorical variables

by state CON laws compared with counties located in states without CON. Relatedly, the proportion of all nursing home beds located in medium- and large-sized facilities was greater in counties subject to $\mathrm{CON}$, while the proportion of nursing home beds located in smaller facilities was higher for counties that were not subject to CON.

The adjusted association between average nursing home size and CON laws with the number of SARS-CoV-2 cases on June 12, controlling for population size and other covariates, is described in Table 2. A between-county 
a

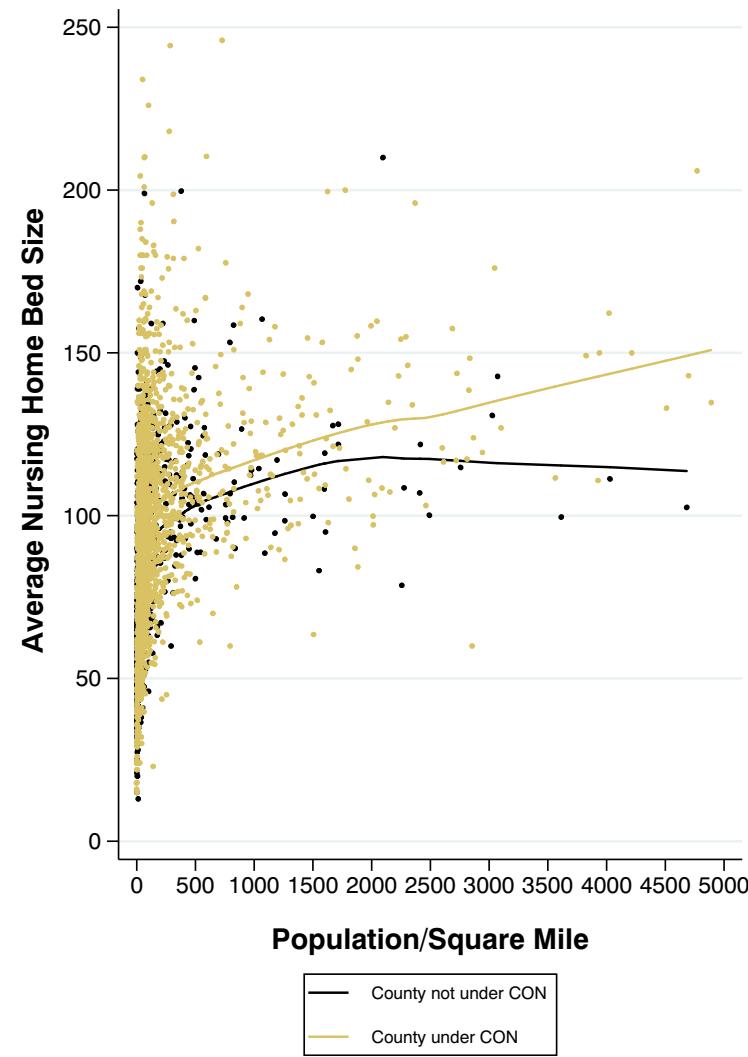

b

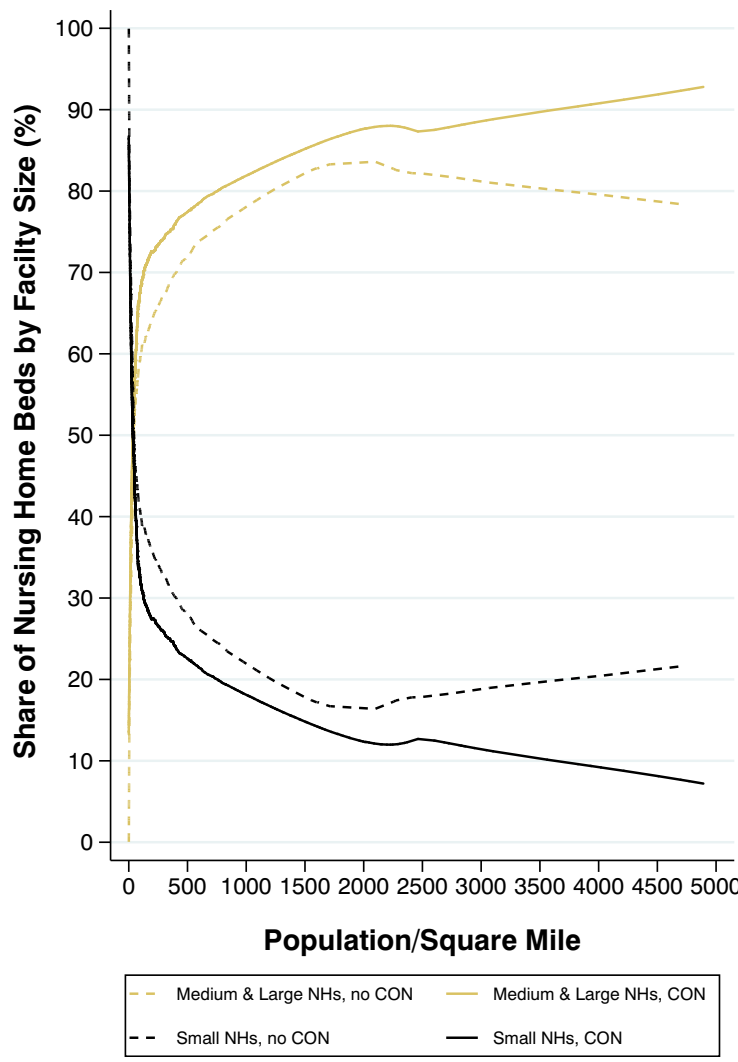

Figure 1 Nursing home size in counties located in states with and without certificate of need laws. (a) Average nursing home size. (b) Proportion of beds in small, medium, and large nursing homes. Notes: $\mathrm{NH}=$ Nursing home; $\mathrm{CON}=$ certificate of need law. Small nursing homes are defined as those with less than 90 beds. Large nursing homes are defined as those with greater than 120 beds. Trend lines were estimated via locally weighted scatterplot smoothing (local linear regression). To improve visual clarity, 11 counties with population densities greater than 5,000 were excluded from the plot.

difference in average nursing home size equal to 1 bed was associated with 3.92 additional SARS-COV-2 cases (95\% CI, 2.14 to 5.69; $P<0.001$ ), on average, and counties subject to CON laws had 104.53 additional SARS-CoV-2 cases (95\% CI, 7.68 to 201.38; $P<0.05$ ), on average. Notably, the associations between most other health care supply measures (e.g., the total number of nursing home beds and the number of home health agencies) with the number of SARS-CoV-2 cases were not statistically significant.

Figure 2 illustrates the relationship between the adjusted count of SARS-CoV-2 cases per 100,000 persons on June 12 with average nursing bed home size at the state level. Average nursing home size varies widely across states from about 45 beds in Alaska to over 175 beds in New York. States with larger nursing homes exhibited higher infection rates and were more likely to be subject to a CON law. Figure 3 shows trends in the adjusted number of SARS-CoV-2 cases by average nursing home size (panel A) and CON law status (panel B). Appendix Figure 1 shows trends in the adjusted number of SARSCoV-2 cases among all counties with and without at least one large nursing home (panel A) and specifically for metropolitan counties (panel B). SARS-CoV-2 growth was larger in counties with an average nursing home bed size of 90 or more beds, counties with at least one large nursing home, and for counties located in states with $\mathrm{CON}$ laws.

\section{Secondary Analyses}

The adjusted association between average nursing home size and CON laws with the number of SARS-CoV-2related deaths on June 12 is described in Appendix Table 1. A between-county difference in average nursing home size equal to 1 bed was associated with 0.18 additional deaths ( $95 \% \mathrm{CI}, 0.7$ to $0.28 ; P<0.001$ ), on average. The presence of a CON law was not associated with SARS-CoV-2-related deaths.

Appendix Table 2 shows the adjusted association between three alternative primary explanatory variables and the number of SARS-CoV-2 cases. In each specification, nursing home size was associated with a greater number of infections. Appendix Table 3 shows the results from a specification including both the interaction between CON status and average nursing home size. In this specification, average nursing home size remained significantly associated with the number of SARS-CoV-2 cases; however, the coefficients for CON status and the interaction 
Table 2 Association of Average Nursing Home Size and Certificate of Need Laws with the Number of SARS-CoV-2 Cases on June 12

\begin{tabular}{|c|c|c|c|c|}
\hline \multirow[b]{3}{*}{ Covariates } & \multicolumn{4}{|c|}{ Outcome: No. SARS-CoV-2 cases } \\
\hline & \multicolumn{2}{|l|}{ Model 1} & \multicolumn{2}{|l|}{ Model 2} \\
\hline & $\beta$ & $95 \% \mathrm{CI}$ & $\beta$ & $95 \% \mathrm{CI}$ \\
\hline Average nursing home size & $3.92 * * *$ & $(2.14,5.69)$ & -- & -- \\
\hline Certificate of need law & -- & -- & $104.53 *$ & $(7.68,201.38)$ \\
\hline Population size (1000s) & -0.37 & $(-1.19,0.45)$ & -0.29 & $(-1.16,0.58)$ \\
\hline Population density & $0.03 * *$ & $(0.01,0.06)$ & $0.04 * * *$ & $(0.02,0.06)$ \\
\hline Metropolitan county & $621.23 * * *$ & $(493.22,749.23)$ & $685.81 * * *$ & $(545.19,826.44)$ \\
\hline Midwest region (ref. Northeast) & $-657.87 * * *$ & $(-945.38,-370.36)$ & $-892.28 * * *$ & $(-1207.56,-577.00)$ \\
\hline South region (ref. Northeast) & $-1043.12 * * *$ & $(-1295.62,-790.63)$ & $-1226.60 * * *$ & $(-1517.95,-935.25)$ \\
\hline West region (ref. Northeast) & $-1054.01 * * *$ & $(-1344.85,-763.18)$ & $-1259.31 * * *$ & $(-1587.43,-931.20)$ \\
\hline$\%$ Aged 65 and over & $-44.72 * * *$ & $(-64.52,-24.92)$ & $-45.96 * * *$ & $(-65.55,-26.38)$ \\
\hline$\%$ Female & $45.01 *$ & $(9.91,80.10)$ & $47.05 * *$ & $(11.41,82.69)$ \\
\hline$\%$ Black & $13.01 * * *$ & $(9.20,16.82)$ & $13.58 * * *$ & $(9.69,17.47)$ \\
\hline$\%$ College educated & -4.62 & $(-10.63,1.38)$ & $-7.19 *$ & $(-13.88,-0.50)$ \\
\hline Median household income (1000s) & $19.05 * * *$ & $(12.31,25.79)$ & $20.99 * * *$ & $(13.8,28.18)$ \\
\hline Poverty rate & $30.02 * * *$ & $(15.6,44.43)$ & $30.51 * * *$ & $(16.07,44.95)$ \\
\hline Nursing home total bed supply & 0.05 & $(-0.02,0.11)$ & 0.05 & $(-0.02,0.12)$ \\
\hline Bed supply, small hospitals & 0.33 & $(-0.17,0.84)$ & 0.33 & $(-0.20,0.85)$ \\
\hline Bed supply, medium-sized hospitals & $-0.16 * *$ & $(-0.26,-0.06)$ & $-0.17 * *$ & $(-0.28,-0.06)$ \\
\hline Bed supply, large hospitals & -0.06 & $(-0.14,0.02)$ & -0.04 & $(-0.13,0.04)$ \\
\hline No. home health agencies & -1.16 & $(-3.10,0.78)$ & -1.57 & $(-3.82,0.69)$ \\
\hline No. medical doctors (per 1000) & -23.77 & $(-66.17,18.62)$ & -35.82 & $(-82.85,11.22)$ \\
\hline No. housing units & $2.69 *$ & $(0.56,4.82)$ & $2.62 *$ & $(0.37,4.87)$ \\
\hline Date of first SARS-CoV-2 Case & $36.03 * * *$ & $(28.48,43.59)$ & $36.73 * * *$ & $(29.07,44.38)$ \\
\hline
\end{tabular}

SARS-CoV-2, severe acute respiratory syndrome coronavirus 2; No., number of. Marginal effects (betas) and 95\% CIs are derived from a Poisson regression model including robust standard errors. In model 1, the main explanatory variable is average nursing home bed size. In model 2 , the main explanatory variable is certificate of need law presence. Population density is equal the number of persons per square mile of land. Small-, medium-, and large-sized hospitals denote those with, $<100,100$ to 399 , and $\geq 400$ beds, respectively

$* P<0.05$

$* * P<0.01$

$* * * P<0.001$

term were not statistically significant. Excluding counties located in New York State and New Jersey also generated results consistent with the primary findings (Appendix Table 4).

\section{DISCUSSION}

In this study, we found that a county's average nursing home size and the presence of a CON law were associated with a greater frequency of SARS-CoV-2 cases over time. By the end of the study period, a one bed between-county difference in average nursing home size was associated with nearly four additional SARS-CoV-2 cases after adjustment for population size and density, overall nursing home bed supply, and a large set of other demographic, socioeconomic, and health care-related covariates. Counties subject to CON laws had on average 104 more SARS-CoV-2 cases than counties not subject to CON. We also found that average nursing home size was associated with SARS-CoV-2-related mortality; however, the magnitude of this association was considerably lower. While the presence of larger nursing homes may accelerate infection rates in a community, mortality rates are more likely to be related to clinical factors.

Our findings are consistent with recent studies showing that nursing home size is a strong predictor of whether a facility experienced a SARS-CoV-2 outbreak. ${ }^{6,7}$ However, we conducted an analysis of SARS-CoV-2 cases in the areas nursing homes are located, which is distinctly important since larger facility size places not only nursing home residents, but also community members at risk of contracting SARS-CoV-2. While the majority of SARS-CoV-2 cases is likely accounted for by the non-nursing home population, our results may indicate the potential role of larger nursing homes as accelerants of community infection rates. In addition, our study includes data from March and April, 2020, a period of time when the majority of nursing homes were not reporting outbreaks publicly. In fact, the Centers for Medicare and Medicaid Services began requiring nursing homes to submit COVID-19-related data in mid-May, and allowed facilities to optionally submit prior data.

Consistent with prior reports, we show that the size of nursing homes depends in part on long-term care policies, and particularly CON laws. ${ }^{10}$ New York State, for instance, has a CON law and its nursing homes are over 1.5 times the size of those in California, a non-CON state with comparable population density. CON laws for nursing homes have been in place for several decades in 37 states and were originally established to limit state Medicaid spending on long-term care by placing a cap on the supply of nursing home beds. However, high nursing home capital costs combined with bed supply ceilings has led to fewer nursing home operators and larger facilities in states with CON. 


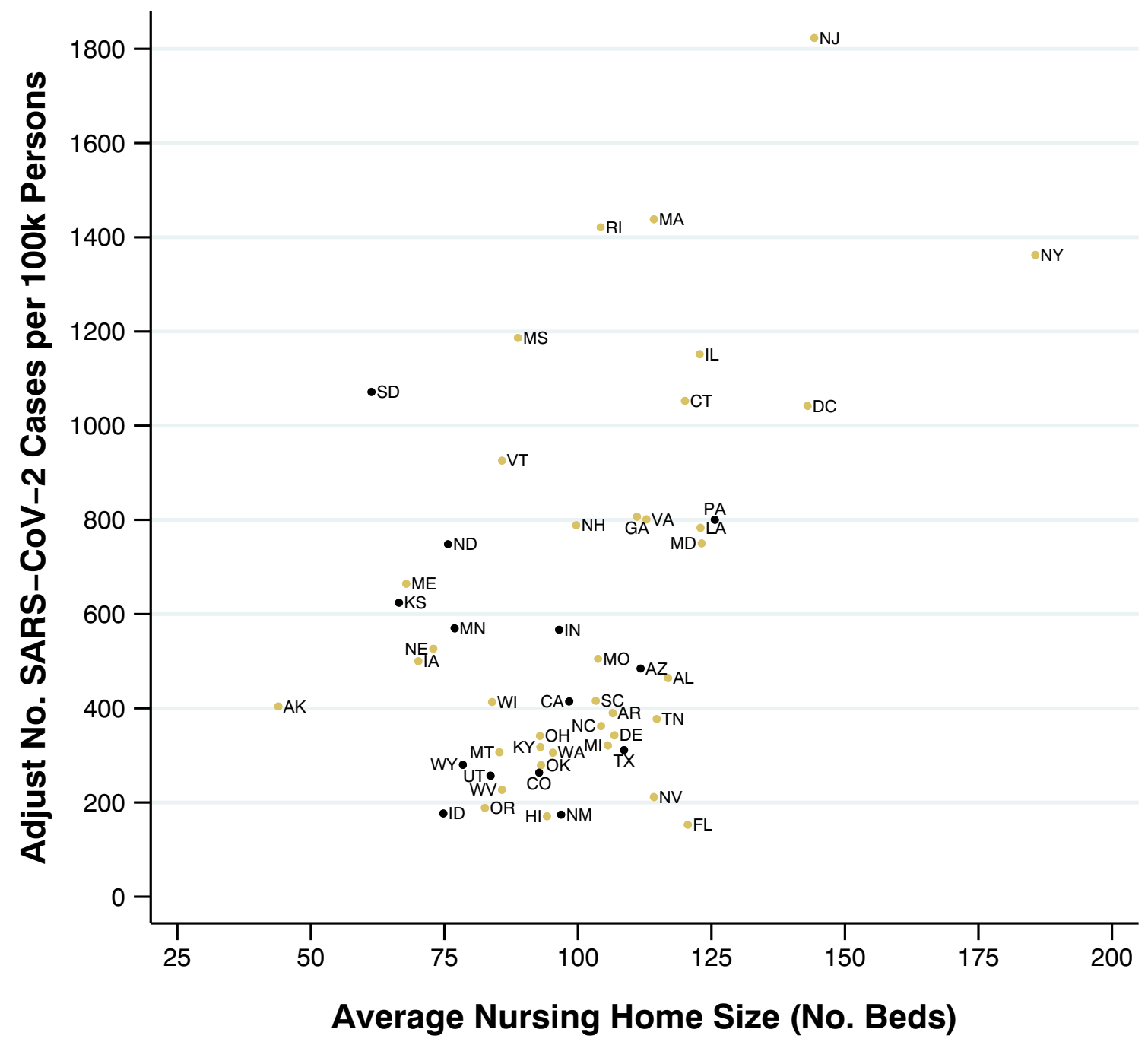

\section{- Under CON LAW - Not under CON Law}

Figure 2 State-level adjusted SARS-CoV-2 prevalence on June 12 by average nursing home size and certificate of need law presence. Notes: SARS-CoV-2, severe acute respiratory syndrome coronavirus 2; No., number of; CON, certificate of need law. Prevalence estimates were derived from a Poisson regression model including robust standard errors. The set of covariates were population size, population density (persons per square land mile), metropolitan versus rural county status, census region, $\%$ of residents aged 65 and over, $\%$ female, $\%$ Black, $\%$ college educated, median household income, the poverty rate, nursing home bed supply, bed supply of small-, medium-, and large-sized hospitals, number of home health agencies, number of medical doctors, number of housing units, and date of the first SARS-CoV-2 case.

Only a few states have repealed their CON laws, potentially because on surface they may appear to be benign. However, there is evidence suggesting that $\mathrm{CON}$ laws were not only ineffective at controlling spending in the long run, but also that nursing home staffing levels have improved at slower rates in states with $\mathrm{CON}$ versus states without $\mathrm{CON} .^{10,11}$ Our findings indicate another potential concern regarding CON laws, which is their association with facility size and disease spread. More generally, these results highlight the potential for long-term care policy consequences outside the long-term care system. Low Medicaid reimbursement, known to be associated with poor sick leave benefits for long-term care works, is an example of another state policy that may impact COVID-19 in the community. ${ }^{16}$

\section{Limitations}

This study has important limitations. First, the analysis was ecological because individual-level COVID-19 data are not currently available. As such, individual-level inferences should not be drawn from these results. The use of aggregate data also precludes measuring the direct contribution of larger nursing homes to community spread, and it is possible that the higher frequency of SARS-CoV-2 cases in counties with larger nursing homes reflects infections among nursing home residents alone. However, it is worth noting that total nursing home bed supply was not significantly associated with the number of SARS-CoV-2 cases. Second, while the SARS-CoV-2 data are widely used, they have not yet been externally validated and the 
a

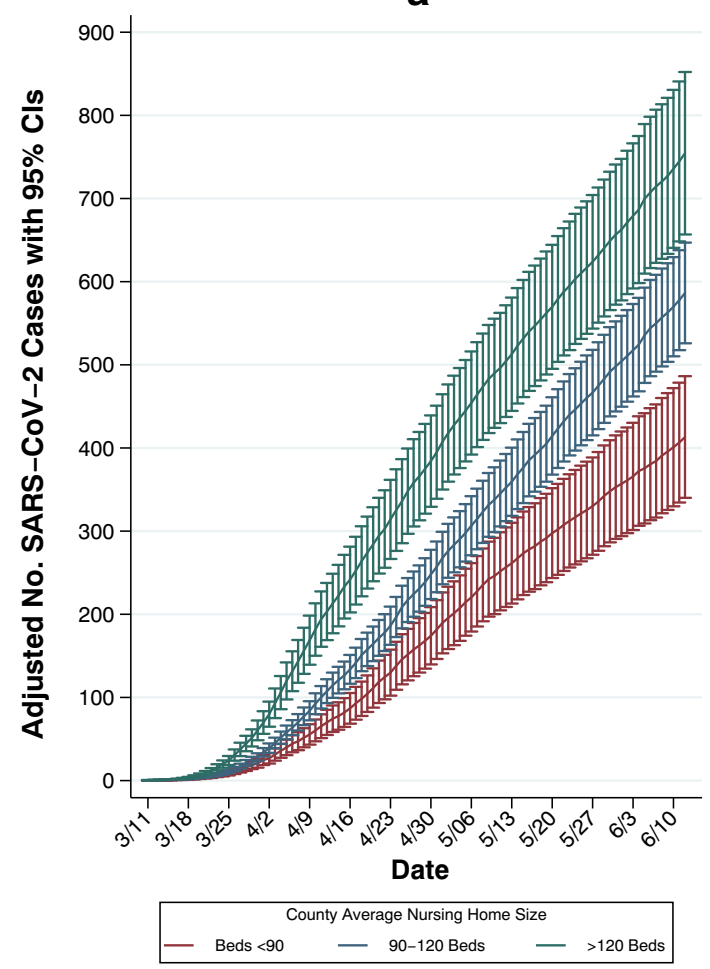

b

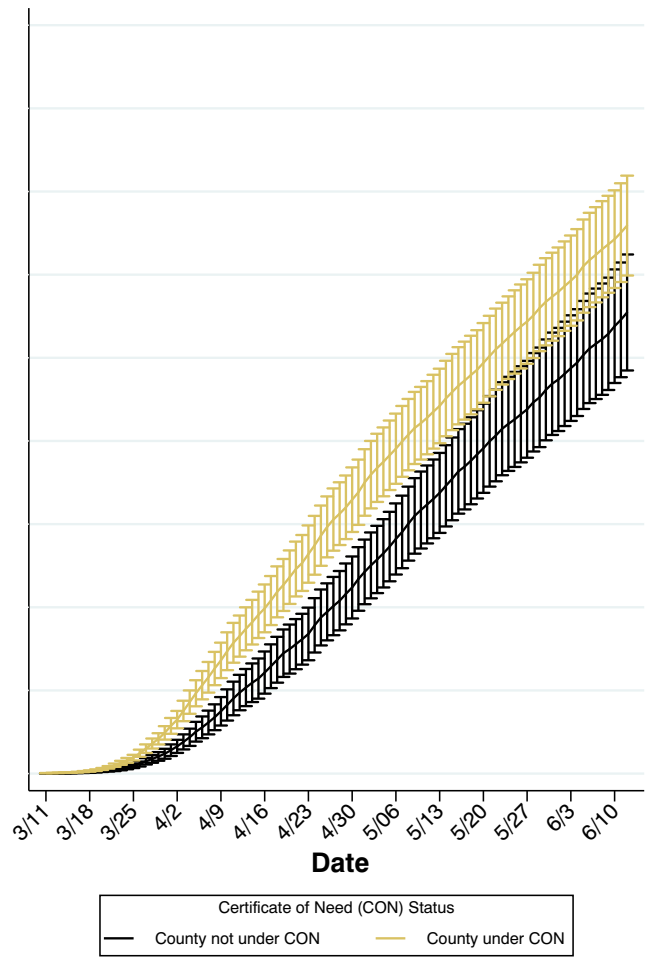

Figure 3 Trends in county-level adjusted SARS-CoV-2 cumulative prevalence by (a) average nursing home size and (b) presence of a certificate of need law. Notes: SARS-CoV-2, severe acute respiratory syndrome coronavirus 2; No., number of; CI, confidence interval. Point estimates and $95 \%$ confidence intervals were derived from a Poisson regression model including robust standard errors. The set of covariates were population size, population density (persons per square land mile), metropolitan versus rural county status, census region, \% of residents aged 65 and over, \% female, \% Black, \% college educated, median household income, the poverty rate, nursing home bed supply, bed supply of small-, medium-, and large-sized hospitals, number of home health agencies, number of medical doctors, number of housing units, and date of the first SARS-CoV-2 case. For panel A, average nursing home size was included in the model (below 90 beds, 90 to 120 beds, 121 ore more beds). For panel $B$, certificate of need law presence was included as a covariate.

quality of the data submitted by public health departments may vary.

Third, due to the cross-sectional design, there remains the potential for residual confounding. For example, social distancing practices, other mobility patterns, and associated local policies were not accounted for and are associated with infection rates. Relatedly, this study does not investigate potential mediating mechanisms underlying the association between nursing home size, CON laws, and SARS-CoV-2 infections. For example, smaller nursing homes may be more readily able to reduce occupancy, be higher quality, or have better access to personal protective equipment. Each of these factors may be related to lower community spread of SARS-CoV-2. Similarly, CON laws may impact attributes of nursing homes other than size, such as staffing levels, which may be associated with SARS-CoV-2 spread. Fourth, although we conducted several robustness checks, our results may be sensitive to other modeling choices. Fifth, while counties are commonly used to approximate health care markets, the size of counties and allocation of nursing homes within them may vary considerably, which introduces noise into the analyses.

In conclusion, at the county level, average nursing home size was associated with a greater populationadjusted count of SARS-CoV-2 cases. Infections were also more frequent in states with certificate of need laws, which influence the size of nursing homes. Controlling the impact of COVID-19 may require additional resources for communities with larger nursing homes and more attention towards long-term care policies.

Supplementary Information The online version contains supplementary material available at https://doi.org/10.1007/ s11606-020-06518-2.

Acknowledgments: Contributors: There were no individuals who contributed to the manuscript and did not meet authorship criteria.

Corresponding Author: Momotazur Rahman, PhD; Department of Health Services, Policy \& Practice, Brown University School of Public Health, Providence, RI, USA (e-mail: momotazur_rahman@brown.edu).

Funding This study was supported by a grant from the US National Institute on Aging (3PO1AG027296-11S1)

\section{Compliance with Ethical Standards:}

Conflict of Interest: The authors were supported by a grant from the National Institute on Aging (3P01AG027296-11S1). The National Institute on Aging had no role in the design and conduct of the study; collection, management, analysis, and interpretation of the data; preparation, review, or approval of 
the manuscript; and decision to submit the manuscript for publication.

\section{REFERENCES}

1. John Hopkins University Coronavirus Resource Center. Coronavirus COVID-19 global cases. https://coronavirus.jhu.edu/map.html. Accessed June 28, 2020.

2. Wadhera RK, Wadhera P, Gaba P, et al. Variation in COVID-19 Hospitalizations and Deaths Across New York City Boroughs. JAMA. 2020;323(21):2192-2195.

3. Millett GA, Jones AT, Benkeser D, et al. Assessing differential impacts of COVID-19 on Black communities. Ann Epidemiol. 2020;47:37-44.

4. Lau-Ng R, Caruso LB, Perls TT. COVID-19 deaths in long term care facilities-a critical piece of the pandemic puzzle. J Am Geriatr Soc. 2020;68(9): 1895-1898.

5. Kaiser Family Foundation. State Data and Policy Actions to Address Coronavirus. Available at: https://www.kff.org/health-costs/issue-brief/ state-data-and-policy-actions-to-address-coronavirus/ Accessed June 20, 2020.

6. Abrams HR, Loomer L, Gandhi A, Grabowski, DC. Characteristics of US Nursing Homes with COVID-19 Cases. J Am Geriatr Soc. 2020;68(8): 1653-1656.

7. White EM, Kosar CM, Feifer RA, et al. Variation in SARS-CoV-2 prevalence in US skilled nursing facilities. $J$ Am Geriatr Soc. 2020;68(10):2167-2173.

8. Van Houtven CH, DePasquale N, Coe NB. Essential long-term care workers commonly hold second jobs and double-or triple-duty caregiving roles. J Am Geriatr Soc. 2020;68(8):1657-1660.
9. Bowblis JR, Smith AC. Occupational licensing of social services and nursing home quality: A regression discontinuity approach. ILR Review. June 2019.

10. Ferdows NB, Rahman M. Evolution of the Nursing Home Industry in States With Different Certificate of Need Policies. J Am Med Dir Assoc. 2020;21(4):559.

11. Rahman M, Galarraga O, Zinn JS, Grabowski DC, Mor V. The impact of certificate-of-need laws on nursing home and home health care expenditures. Med Care Res Rev. 2016;73(1):85-105.

12. [dataset] AHRF Health Resources and Services Administration. Area Health Resources Files (AHRF). 2014. https://datawarehouse.hrsa.gov/ topics/ahrf.aspx

13. [dataset] COVID-19 Data Repository by the Center for Systems Science and Engineering (CSSE) at Johns Hopkins University. 2020. https:// github.com/CSSEGISandData/COVID-19.

14. Wu X, Nethery RC, Sabath BM, et al. Exposure to air pollution and COVID-19 mortality in the United States. medRxiv [Preprint]. 2020 [cited 2020 June 20]: Available from: https://www.medrxiv.org/content/medrxiv/early/2020/04/27/2020.04.05.20054502.full.pdf

15. US Department of Agriculture. Rural-Urban Continuum Codes. https:// www.ers.usda.gov/data-products/ruralurban-continuum-codes/.aspx

16. Grabowski DC. Strengthening Nursing Home Policy for the Postpandemic World: How Can We Improve Residents' Health Outcomes and Experiences? Available at: https://www.commonwealthfund.org/sites/default/ files/2020-08/Grabowski_strengthening_nursing_home_policy_postpandemic_ib.pdf. Published August, 2020. Accessed October 7, 2020.

Publisher's Note: Springer Nature remains neutral with regard to jurisdictional claims in published maps and institutional affiliations. 